\title{
Cluster methods for the description of a driven-dissipative spin model
}

\author{
D. Huybrechts* and M. Wouters \\ TQC, Universiteit Antwerpen, Universiteitsplein 1, B-2610 Antwerpen, Belgium
}

(Received 30 November 2018; published 29 April 2019)

\begin{abstract}
We study the influence of short-range quantum correlations and classical spatial correlations on the phase diagram of the dissipative $X Y Z$ model by using a Gutzwiller Monte Carlo method and a cluster Gutzwiller ansatz for the wave function. Considering lattices of finite size we confirm the emergence of a region with ferromagnetic correlations and two paramagnetic regions and show the emergence of a region with a buildup of ferromagnetic correlations completely missed by mean-field theory. The inclusion of short-range quantum correlations causes drastic alterations of the mean-field correlation functions but our results show that the inclusion of long-range quantum correlations or the use of more sophisticated methods is needed to quantitatively match the exact results. A study of the susceptibility tensor shows that reciprocity is broken, a feature not observed in closed quantum systems. Furthermore, increasing the magnetic field suppresses the magnetization; this is also in contrast with closed quantum systems.
\end{abstract}

DOI: 10.1103/PhysRevA.99.043841

\section{INTRODUCTION}

In recent years the field of dissipative phase transitions has been under intense study due to technological advances which have given access to a wide range of systems where it is possible to study the quantum dissipative behavior of open quantum systems. The experimental platforms include trapped ions, cold atoms, semiconductor microcavities, and cavity and circuit QED [1-4]. Driven-dissipative many-body systems find the origin of their interesting dynamics in the interplay of coherent driving and dissipation. Typically, in the long-time limit, the system is driven into a steady state that is determined by the competition between both Hamiltonian and dissipative dynamics, resulting in a wide range of possible phases. Dissipative phase transitions have been observed in a wide range of experiments [5-11]. Consequently, the interest in the study of dissipative phase transitions has spiked [12-22].

Analytical solutions for open quantum systems are scarce, and if one wants to calculate the dynamics of the system, one has to rely on numerical tools. As usual, a numerically exact description is unfeasible due to the exponential scaling of the Hilbert-space dimension with system size and thus approximations are needed. Different approaches have been applied to a range of open quantum systems. Among them are mean-field approximations [15-19,22-26]; approaches based on matrix product operators and matrix product states [27-35], which have proven to be of great success in one dimension; the corner space renormalization method [20,36]; variational methods for the master equation [21,37-41]; and variational methods at the level of the wave function [42-47].

In this work we study the dissipative $X Y Z$ Heisenberg model. Previous works have investigated the steady-state phase diagram by using a mean-field approach [22], a

\footnotetext{
*dolf.huybrechts@uantwerpen.be
}

cluster mean-field approach (CMF) [23], and the corner space renormalization method [20]. The CMF has also been used together with a self-consistent Mori projector approach to investigate the (non)existence of limit cycles [26]. A tensor network algorithm [48] and a driven-dissipative quantum Monte Carlo [47] have also been applied to this model. In Ref. [43] this model and its phase transitions are studied using quantum trajectories and a single-site Gutzwiller ansatz using the Gutzwiller Monte Carlo approach (GMC). In this work we extend this approach by applying a cluster Gutzwiller ansatz for the wave function. We refer to this method as the cluster Gutzwiller Monte Carlo approach (CGMC). By including quantum correlations we show the possible existence of a dissipative phase transition, not predicted by the classical mean-field method, through studying the steady-state spin structure factor and the susceptibility.

We introduce the model we work with in Sec. II. The method of quantum trajectories, the used unraveling, and the cluster Gutzwiller ansatz are explained in Sec. III. In Sec. IV we discuss the steady-state spin structure factor. The influence of an applied magnetic field is studied in Sec. V. In Sec. VI the susceptibility and the possible existence of a new phase transition are looked into. Finally, conclusions are formulated in Sec. VII.

\section{THE MODEL SYSTEM}

We study the anisotropic $X Y Z$ Heisenberg Hamiltonian (with $\hbar=1$ ) on a spin lattice,

$$
\hat{H}=\sum_{\langle i, j\rangle}\left(J_{x} \hat{\sigma}_{i}^{(x)} \hat{\sigma}_{j}^{(x)}+J_{y} \hat{\sigma}_{i}^{(y)} \hat{\sigma}_{j}^{(y)}+J_{z} \hat{\sigma}_{i}^{(z)} \hat{\sigma}_{j}^{(z)}\right),
$$

where $J_{x}, J_{y}$, and $J_{z}$ are the coupling strengths in the $x, y$, and $z$ directions, $\hat{\sigma}_{i}^{\alpha}$ the Pauli matrices $(\alpha=x, y, z)$, and the sum goes over the nearest neighbors in the lattice. Periodic boundary conditions are applied at the edge of the lattice. A proposal for an experimental setup based on the optical 
pumping of two-level atoms has been made in Ref. [22], resulting in the above Hamiltonian with effective spins. This Hamiltonian governs the unitary part of the time evolution of the system. The total time evolution of the system is governed by a Lindblad equation with dissipation along the $z$ axis:

$$
\partial_{t} \hat{\rho}=-i[\hat{H}, \hat{\rho}]+\frac{\gamma}{2} \sum_{j}\left(2 \hat{\sigma}_{j}^{(-)} \hat{\rho} \hat{\sigma}_{j}^{(+)}-\left\{\hat{\sigma}_{j}^{(+)} \hat{\sigma}_{j}^{(-)}, \hat{\rho}\right\}\right) .
$$

With $\gamma$ the decay rate of the spins and $\hat{\sigma}_{i}^{(+)}\left[\hat{\sigma}_{i}^{(-)}\right]$the raising [lowering] operators along the $z$ axis. This driven-dissipative Heisenberg model has recently been the subject of several studies attempting to describe the dynamics. In this work we compare our results with the CMF [23]. We consider the parameters $J_{x}=0.9 \gamma$ and $J_{z}=\gamma$, unless stated differently, and vary $J_{y}$. The mean-field approach [22] predicts a transition from a paramagnetic phase to a ferromagnetic phase for this parameter set at $J_{y} \approx 1.04 \gamma$. Upon including quantum correlations in the CMF [23] or classical spatial correlations in the GMC [43], the existence of another transition from the ferromagnetic phase to the paramagnetic phase is observed.

\section{QUANTUM TRAJECTORIES AND THE WAVE FUNCTION ANSATZ}

The theory of quantum stochastic processes was first introduced by Davies [49] and has been further developed [50-55] into the quantum trajectory formalism, also known as the Monte Carlo wave function method. It offers an alternative to calculating the dynamics of the system with a Lindblad equation for the density matrix of the open system. Instead of solving for the density matrix, the dynamics of the system are calculated on the level of the wave function. Through a stochastic process, many realizations of the wave function, so-called quantum trajectories, are simulated, and by averaging over these trajectories one recovers the dynamics of the open quantum system. This method is often referred to as the unraveling of the master equation. The stochastic process finds its origin in the continuous measurement of the environment. This continuous measurement results in random changes of the wave function of the system. The unraveling of the master equation is not unique, as there are several possible measurements of the environment. In this work we unravel the master equation through the process of photon counting [56]. Usually the environment is under constant observation for emitted photons in this measurement scheme. As we are working with a spin system we do not count the excitations of the environment caused by emitted photons; we do, however, monitor the environment for excitations caused by spin flips in the system. In between the detection of these excitations the wave function will evolve according to

$$
\psi(t)=\frac{\exp (-i H t) \tilde{\psi}}{\|\exp (-i H t) \tilde{\psi}\|}
$$

with $\tilde{\psi}$ an initial (normalized) wave function. Note that the time evolution generated by

$$
H=\hat{H}-i \frac{\gamma}{2} \sum_{i} \hat{\sigma}_{i}^{(+)} \hat{\sigma}_{i}^{(-)}
$$

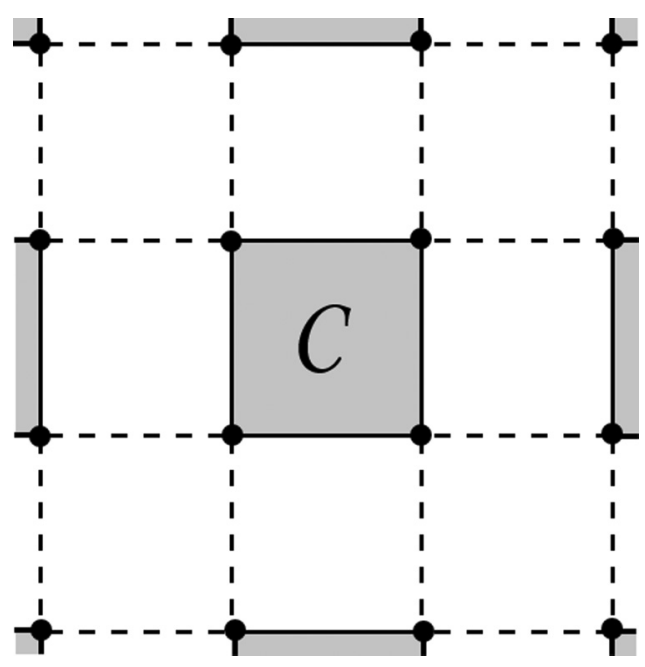

FIG. 1. Lattice layout with $2 \times 2$ clusters on a 2D lattice. Each cluster is shown as a gray area and contains a set of ('connected') lattice points. Inside these clusters quantum correlations between the different sites are included.

does not preserve the norm. When an excitation is detected a quantum jump is made in the evolution of the wave function,

$$
\psi \rightarrow \frac{\hat{\sigma}_{i}^{(-)} \psi}{\left\|\hat{\sigma}_{i}^{(-)} \psi\right\|}
$$

after which the wave function continues evolving according to (3).

Numerically solving the master equation for the solution of the density matrix is computationally very demanding. Exact solutions are unfeasible already for small systems. One of the reasons for this inconvenient characteristic of open manybody systems is the exponentially large Hilbert space. This is where one makes some efficiency gain by using quantum trajectories: in the master equation approach one has to work with the quadratically large Hilbert space $\mathcal{H}^{2}$ of the density matrix, whereas in the trajectory approach one works with the Hilbert space $\mathcal{H}$ of the wave function. This advantage comes at the cost of needing to average over multiple realizations to obtain the dynamics of the open system. The number of realizations needed, however, is usually much smaller than the dimension of the Hilbert space.

The quantum trajectory method, however, does not solve the exponential scaling of computational resources with system size. In order to reduce the dimensionality of the problem, an ansatz for the wave function is considered. Previous work has investigated the Gutzwiller ansatz [57] applied to the dissipative XYZ Heisenberg model [43]. We extend this approach by including short-range quantum correlations through the use of the cluster Gutzwiller ansatz. This ansatz is realized by considering a sublattice of clusters, as shown in Fig. 1, where each cluster contains a set of (nearest-neighbor) lattice points

$$
\Psi_{\mathrm{GW}}(\{\mathcal{C}\})=\prod_{\mathcal{C}} \psi_{\mathcal{C}},
$$

where the product runs over the different clusters $\mathcal{C}$ with cluster wave function $\psi_{\mathcal{C}}$. This approach allows for the inclusion of both classical and quantum correlations inside the clusters 
and only classical correlations between the clusters. The time evolution of $\Psi_{\mathrm{GW}}$ can now be determined by calculating the time evolution of the individual cluster wave functions $\psi_{\mathcal{C}}$ according to (3). The non-Hermitian Hamiltonian $H$ for a certain cluster $\mathcal{C}$ in the mean-field approximation is given by

$$
H_{\mathcal{C}}=\hat{H}_{\mathcal{C}}+\hat{H}_{\mathcal{B}(\mathcal{C})}-i \frac{\gamma}{2} \sum_{i \in \mathcal{C}} \hat{\sigma}_{i}^{(+)} \hat{\sigma}_{i}^{(-)},
$$

where $\hat{H}_{\mathcal{C}}$ only contains interactions within the cluster $\mathcal{C}$, given by

$$
\hat{H}_{\mathcal{C}}=\sum_{\alpha=x, y, z} \sum_{\langle i, j\rangle \mid i, j \in \mathcal{C}} J_{\alpha} \hat{\sigma}_{i}^{(\alpha)} \hat{\sigma}_{j}^{(\alpha)} .
$$

The term $\hat{H}_{\mathcal{B}(\mathcal{C})}$ describes the interactions along the boundary $\mathcal{B}$ of cluster $C$, i.e., the interactions between sites inside the cluster and their nearest neighbors outside this cluster, also called the mean-field interactions. This term is given by

$$
\hat{H}_{\mathcal{B}(\mathcal{C})}=2 \sum_{\alpha=x, y, z} \sum_{\langle i, j\rangle \mid i \in \mathcal{C}, j \notin \mathcal{C}} J_{\alpha} \hat{\sigma}_{i}^{(\alpha)}\left\langle\hat{\sigma}_{j}^{(\alpha)}\right\rangle
$$

It is clear that this results in a system of coupled equations for every cluster wave function $\psi_{\mathcal{C}}$, as the time evolution of $\psi_{\mathcal{C}}$ depends on expectation values with respect to the neighboring clusters. To keep the dimension of the Hilbert space limited, only small clusters are studied and the importance of shortrange quantum correlations is determined.

\section{STEADY-STATE SPIN STRUCTURE FACTOR}

In order to investigate the dissipative phase transition between a paramagnetic and a ferromagnetic state, we consider the steady-state spin structure factor $S_{S S}^{x x}(\mathbf{k}=0)$, where

$$
S_{S S}^{x x}(\mathbf{k})=\frac{1}{N(N-1)} \sum_{j \neq l} e^{i \mathbf{k} \cdot(\mathbf{j}-\mathbf{l})}\left\langle\hat{\sigma}_{j}^{(x)} \hat{\sigma}_{l}^{(x)}\right\rangle .
$$

We use this correlation function rather than the spontaneous magnetization itself, because in a finite system, the $\mathbb{Z}_{2}$ symmetry does not spontaneously break. Alternatively, a (small) magnetic field could be applied to break the symmetry, as discussed in Sec. V.

A nonzero value of the steady-state spin structure factor indicates a region with ferromagnetic correlations. A zero value has a wider range of possibilities such as a paramagnetic region, an antiferromagnetic region, and spin density waves. To distinguish between these regions different values of the wave vector have to be studied.

We simulate the dynamics of a trajectory over a minimum total time of $10000 / \gamma$ and obtain the steady-state solutions by time averaging over this single trajectory. Generally, the number of independent points in this time window is of the same order as the length of the time window, i.e., proportional to 10000 . However, at certain points in the phase diagram, for example, near a transition between regions or at certain cluster sizes, the need to use larger time windows may arise. This is because the time for two points to become uncorrelated, and thus independent, may be larger in these cases and the time window thus contains fewer independent points. Increasing the time window by a factor of 2 to 5 is usually sufficient to obtain a good accuracy. The results of our numerical

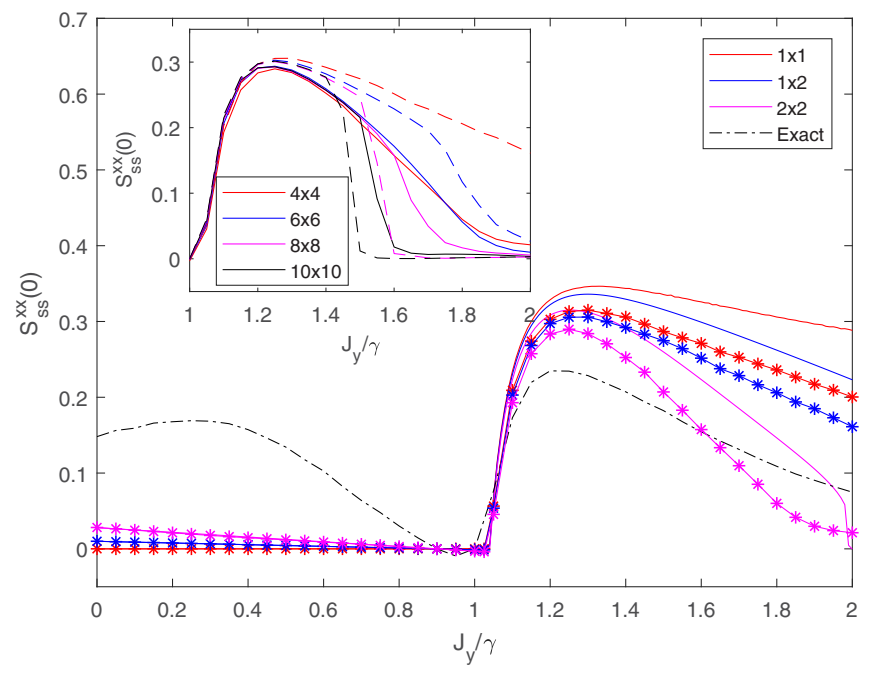

FIG. 2. Steady-state spin structure factor of a $4 \times 4$ lattice with $1 \times 1$ clusters (first top-right line), $1 \times 2$ clusters (second top-right line), and $2 \times 2$ clusters (third top-right line) for the CGMC (asterisks) and the CMF (solid lines). The dash-dotted line is the exact solution of the $4 \times 4$ lattice obtained by using quantum trajectories for the time evolution of the full Hilbert space. Note that the $1 \times 1$ CMF is the usual mean-field result. The CGMC qualitatively predicts the same ferromagnetic and two paramagnetic regions as the CMF [25]. Additionally, we observe the possible existence of a phase transition completely missed by mean-field theory for values of $J_{y}<$ $0.9 \gamma$. Inset: Steady-state spin structure factor of $4 \times 4$ (first top-right line), $6 \times 6$ (second top-right line), $8 \times 8$ (third top-right line), and $10 \times 10$ (fourth top-right line) lattices with clusters of size $1 \times 2$ (dashed lines) and $2 \times 2$ (solid lines) for the CGMC. Increasing the lattice size shows a sharpening of the transition, also found with the GMC [43]. This sharpening is steeper when larger cluster sizes are included.

simulations are shown in Fig. 2, where the spin structure factor was obtained for a $4 \times 4$ lattice with various cluster sizes. It is clear that increased incorporation of quantum correlations present for larger cluster sizes significantly affects the spin correlations.

Both the $1 \times 2$ and the $2 \times 2$ clusters show the existence of the ferromagnetic region and show qualitatively the same behavior as predicted by the CMF [25] and the GMC [43]. The clusters, however, are able to find a nonzero value for $S_{s s}^{x x}(0)$ for values of $J_{y}<0.9 \gamma$. This behavior is not captured by the single-site Gutzwiller ansatz [43] or the mean field [22] and thus originates from quantum correlations. In the GMC [43] the ferromagnetic region becomes smaller with growing system size and the transition to the paramagnetic region becomes sharper. The inset in Fig. 2 shows that by including clusters of size $1 \times 2$ and $2 \times 2$ this behavior is captured already for smaller lattice sizes. Increasing the cluster size makes the sharpening become steeper and occur for smaller values of $J_{y}$. This shows the importance of quantum correlations in the simulation of an open quantum system. This sharpening is what is to be expected when the size of the lattice grows (i.e., when the thermodynamic limit is approached). 
By comparing the results for different cluster sizes with the exact solution of this lattice we see that for increasing cluster sizes the exact solution is approached more closely, but differences persist. It has to be noted that short-range quantum correlations are not enough to accurately approximate the exact solution for the $4 \times 4$ system. It remains to be seen whether this remains true for larger lattices and whether longer-range quantum correlations have to be taken into account as well.

As mentioned earlier, for values of the parameter $J_{y}$ smaller than $0.9 \gamma$ we find an unexpected buildup of spin-spin correlations. Where the mean-field theory predicts an all-zero steady-state spin structure factor we find a nonzero value by including clusters. This nonzero value is most pronounced in the exact solution. This behavior is completely neglected by the classical mean-field theory and thus entirely driven by quantum fluctuations. The question remains whether or not a phase transition is present. It is, however, clear that short-range quantum correlations do not capture the exact behavior for small lattices, and long-range correlations have to be included for a more accurate description.

A comparison with the CMF used in Ref. [23] can show us the importance of spatial correlations between the clusters, as they are not captured by the CMF. Figure 2 shows the steady-state spin structure factor for several cluster sizes on a $4 \times 4$ lattice. The exact solution is found using the quantum trajectory approach. We note two distinct areas, again for $J_{y}<0.9 \gamma$ and $J_{y}>0.9 \gamma$ (we call them the left-hand and right-hand side, respectively). On the left-hand side both the CMF and the CGMC match closely, giving a strong indication that only quantum correlations are important in this regime. On the right-hand side of the figure, however, the CMF and CGMC match only qualitatively; in this regime both quantum and classical spatial correlations contribute. This confirms the difference in the buildup of order on the left-hand versus the right-hand side, respectively.

In one dimension we can take clusters of a larger linear size. In the top panel in Fig. 3 we look at a $1 \times 12$ lattice for which we can go up to $1 \times 6$ clusters. As was the case for the two-dimensional (2D) lattice, the exact solution was obtained using the quantum trajectory approach. We find that the behavior of the steady-state spin structure factor is qualitatively captured by the cluster approach in both the left and the right region for $1 \times 6$ clusters. It must be noted that the system has no phase transition in the thermodynamic limit in one dimension, which has been shown by using a matrix product operator ansatz for the density matrix [23]. The behavior of the spin structure factor for finite-size systems does, however, give insight into the importance of the longerrange quantum correlations to describe the exact dynamics of the open quantum system. As one can see in Fig. 3, decreasing the size of the clusters results in a steady-state spin structure factor that differs completely from the exact value; even negative values are found for values of $J_{y}>\gamma$ where they should be positive. For values of $J_{y}<\gamma$ the influence of increasing the cluster size can be clearly observed. By including longer-range quantum correlations the exact behavior is matched more closely. For $1 \times 2$ clusters we find the same linear behavior for the steady-state spin structure factor as found in the 2D case. By increasing the cluster size we can see

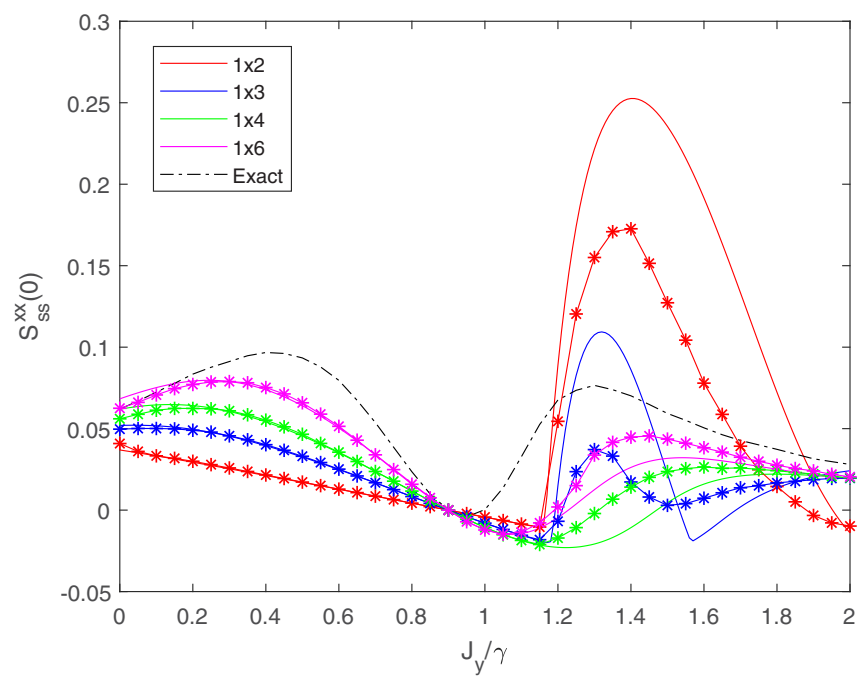

FIG. 3. Steady-state spin structure factor of a $1 \times 12$ lattice with different cluster sizes using the CGMC (asterisks) and the CMF (solid lines). The dash-dotted line represents the exact solution of the $1 \times 12$ lattice obtained by using quantum trajectories for the time evolution of the full Hilbert space. The figure shows that through inclusion of longer-range quantum correlations, by increasing the cluster size, the exact behavior is approached more closely for $J_{y}<$ $\gamma$; we observe a convergence from the result with $1 \times 2$ clusters (bottom asterisk line) up to the result with $1 \times 6$ clusters (top asterisk line) to the exact result. Increasing the cluster size for values of $J_{y}>\gamma$ does not show a clear convergence pattern to the exact solution. The results for $1 \times 6$ clusters do, however, match the exact result most closely. These findings, for both $J_{y}<\gamma$ and $J_{y}>\gamma$, indicate that short-range quantum correlations are not sufficient for the description of the system.

a clear convergence to the same behavior as the exact solution. Short-range quantum correlations are, as such, not sufficient for the description of the system, and longer-range quantum correlations play an important role.

To gain a better understanding of the ferromagnetic region missed by mean-field theory we show the steady-state spin structure factor for the exact solution of $2 \times 2,3 \times 3$, and $4 \times 4$ lattices in the top panel in Fig. 4. The solution of the $2 \times 2$ and $3 \times 3$ lattices was found by solving the master equation and the $4 \times 4$ solution was obtained with the trajectory approach. As shown in this figure, and as expected for finitesize systems, the region where the phase transition occurs is smoothed out and one could suspect that there is only a continuous change of the order parameter rather than an actual phase transition. In the parameter region $\left.J_{y} / \gamma \in\right] 0.9,1$ [, however, the spin structure factor does decrease when the lattice size is increased. (Note that the steady-state spin structure factor is always 0 for the values $J_{y}=J_{x}$ and $J_{y}=J_{z}$ due to the unitary dynamics conserving, respectively, the magnetization in the $z$ direction and $x$ direction [23]). In order to check for the convergence as a function of increasing system size, we show in the bottom panel in Fig. 4 the behavior as a function of the system size, together with a fit to the power-law dependence $S_{s s}^{x x}(0, L)=a L^{b}$, where $L$ is the number of points along one dimension of the $L \times L$ lattice. In all cases, we find a negative exponent $b$, which is compatible with a vanishing of the spin 

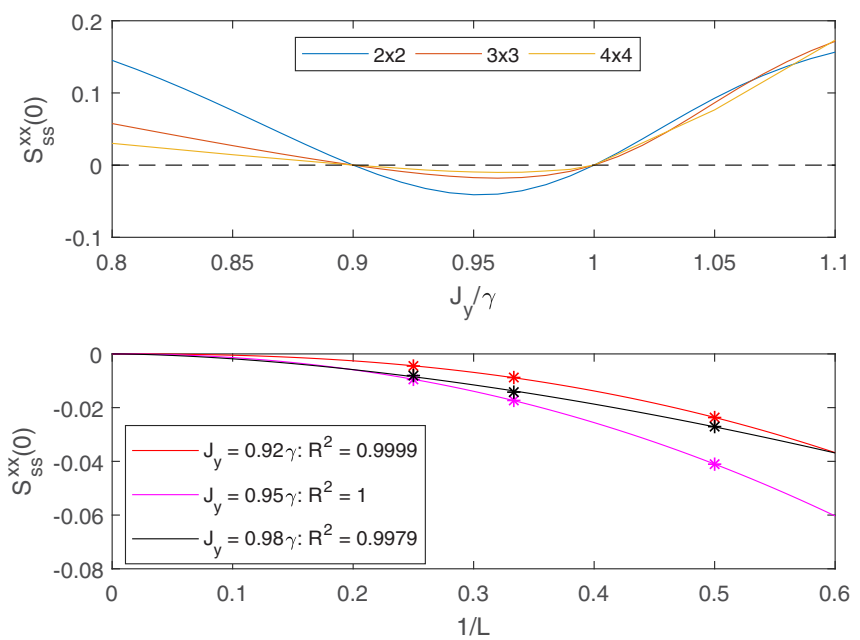

FIG. 4. Top: Exact solution for the steady-state spin structure factor of $2 \times 2,3 \times 3$, and $4 \times 4$ lattices. To exclude the possibility of the presence of a continuous variation of the order parameter rather than a phase transition driven by quantum correlations, we study whether the steady-state spin structure factor in the region $\left.J_{y} \in\right] J_{x}, J_{z}[=] 0.9 \gamma, \gamma[$ converges to 0 in the thermodynamic limit. The solution of the $2 \times 2$ and $3 \times 3$ lattices was found by solving the master equation and the $4 \times 4$ solution was obtained with the trajectory approach. Bottom: Behavior of the steady-state spin structure factor through a fit as a function of the lattice size from the known points of the $2 \times 2,3 \times 3$, and $4 \times 4$ lattices for several values of $\left.J_{y} \in\right] J_{x}, J_{z}\left[: J_{y}=0.92 \gamma\right.$ (top line), $J_{y}=0.95 \gamma$ (bottom line), and $J_{y}=0.98 \gamma$ (middle line). The fit of a power law of the form $S_{s s}^{x x}(0, L)=a L^{b}$ for $L \times L$ lattices returns high $R^{2}$ values and converges to 0 in the thermodynamic limit.

structure factor in the thermodynamic limit. For $J_{y}=0.95 \gamma$ (in the middle of the interval), we find $b \approx-2.1$, close to the value $b=-2$, which is expected for a $2 \mathrm{D}$ system with a finite correlation length.

\section{AN APPLIED MAGNETIC FIELD}

The $\mathbb{Z}_{2}$ symmetry can be explicitly broken by applying a small magnetic field. In this section we study the behavior of the magnetization of the system as a function of the applied field in the $x$ direction and $y$ direction. An applied field $\vec{h}=$ $h_{x} \vec{e}_{x}+h_{y} \vec{e}_{y}$ translates in adding a term $\hat{H}_{B}$ to the Hamiltonian $\hat{H}$ from (1):

$$
\hat{H}_{B}=h \sum_{i}\left(\cos (\theta) \hat{\sigma}_{i}^{(x)}+\sin (\theta) \hat{\sigma}_{i}^{(y)}\right) .
$$

In Fig. 5 the magnetization in the $y$ direction is shown for a $4 \times 4$ lattice with $1 \times 2$ and $2 \times 2$ clusters as a function of $h_{y}\left(h_{x}=0\right)$ in the CMF. From the theory of closed quantum systems one would expect the magnetization to saturate at \pm 1 . This, however, is not the case as shown in the figure, both the $x$ and the $y$ magnetization go to 0 when the field is increased. To obtain a closer understanding of this behavior we note that it is also present in the mean-field approximation, for which we can find analytic expressions. These expressions will enable us to shed light on this behavior.

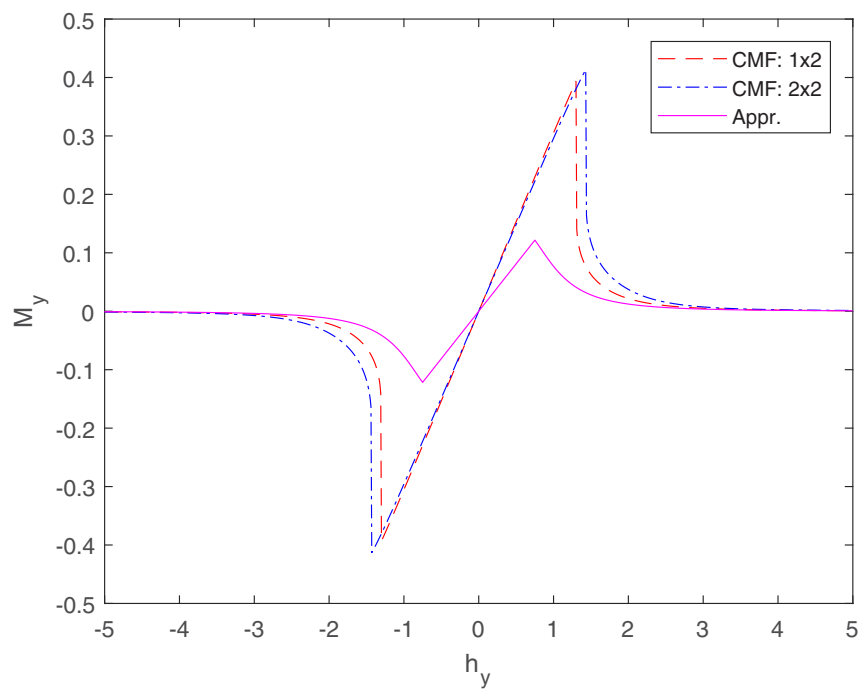

FIG. 5. Magnetization in the $y$ direction as a function of an applied magnetic field in the $y$ direction for $1 \times 2$ (dashed red line) and $2 \times 2$ (dash-dotted blue line) clusters in the CMF and the approximated mean-field solution (solid purple line), which is valid in the limit of large $h_{y}$ for $J_{y}=0.25 \gamma$.

The system of mean-field equations in the steady state can be written as

$$
\begin{aligned}
-\frac{\gamma}{2} M_{x}+2 d\left(J_{y}-J_{z}\right) M_{y} M_{z}+2 h_{y} M_{z} & =0, \\
-\frac{\gamma}{2} M_{y}+2 d\left(J_{z}-J_{x}\right) M_{x} M_{z}-2 h_{x} M_{z} & =0, \\
-\gamma\left(M_{z}+1\right)+2 d\left(J_{x}-J_{y}\right) M_{x} M_{y} & \\
+2\left(h_{x} M_{y}-h_{y} M_{x}\right) & =0,
\end{aligned}
$$

with $d$ the dimensionality, $\gamma$ the dissipation rate, and $M_{x}$, $M_{y}$, and $M_{z}$, respectively, the magnetization in the $x, y$, and $z$ directions. We look at the case where $h_{y} \neq 0$ and $h_{x}=0$ (the reverse situation is analog). With these parameters one can rewrite the system of equations as an expression for $M_{x}$ and $M_{y}$ in terms of $M_{z}$,

$$
\begin{gathered}
M_{x}=\frac{1}{\gamma} \frac{4 h_{y} M_{z}}{1-\frac{16 d^{2}}{\gamma^{2}}\left(J_{y}-J_{z}\right)\left(J_{z}-J_{x}\right) M_{z}^{2}}, \\
M_{y}=\frac{4 d}{\gamma}\left(J_{z}-J_{x}\right) M_{x} M_{z},
\end{gathered}
$$

and an equation for $M_{z}$ whose solution remains to be found by substituting the above solutions for $M_{x}$ and $M_{y}$ into the last equation in (12). This equation has no analytic solution and has to be solved numerically. We can, however, use the knowledge that $M_{z} \rightarrow 0$ as $h_{y} \rightarrow \infty$. A closer study shows that for a growing field $h_{y}, h_{y} M_{z} \rightarrow 0$. With these conditions we can approximate the third equation of (12) up to order $M_{z}$. We then find for large $h_{y}$

$$
M_{z}=-\frac{1}{8} \frac{\gamma^{2}}{h_{y}^{2}} .
$$

This relation explains why $M_{x}$ and $M_{y}$ go to 0 for big applied fields, rather than \pm 1 . This is shown in Fig. 5 as the magenta line. Unlike at thermal equilibrium, the magnetization goes 
to 0 for a large magnetic field. The reason is that in the limit $\left|h_{y}\right| \rightarrow \infty$, the Zeeman term dominates the Hamiltonian, so that the eigenstates are simply the eigenstates of $\sigma_{y}$. The dissipation being in the orthogonal direction does not drive the system to the ground state but, rather, destroys the coherence between the eigenstates. The resulting steady state is then the unit matrix, from which the zero magnetization follows.

For the special case of $J_{x}=J_{y}=J_{z}(\gamma=1)$ the system of mean-field equations is analytically solvable without any approximations:

$$
\begin{aligned}
& M_{x}=-\frac{h_{y}}{\frac{1}{4}+2\left(h_{x}^{2}+h_{y}^{2}\right)}, \\
& M_{y}=\frac{h_{x}}{\frac{1}{4}+2\left(h_{x}^{2}+h_{y}^{2}\right)}, \\
& M_{z}=-\frac{1}{1+8\left(h_{x}^{2}+h_{y}^{2}\right)} .
\end{aligned}
$$

This is in agreement with the above result and is also true for small $h_{x}$ and $h_{y}$. We can conclude that a large magnetic field will cause the system to have no magnetization at all.

Note the difference in sign between $M_{x}$ and $M_{y}$ in this special case. This sign difference implicates that we cannot interchange $x$ and $y$ without introducing a sign change. At first sight this might appear confusing because of the identical parameters $J_{x}=J_{y}=J_{z}$. Only the $z$ direction is fixed by the dissipation and so there appears to be no clear reason for a distinction between $x$ and $y$. A closer look shows that one cannot interchange $x$ and $y$ because this changes the handedness of our coordinate system. This is reflected in the commutation relations of the Pauli matrices requiring that $\left[\hat{\sigma}^{(a)}, \hat{\sigma}^{(b)}\right]=2 i \epsilon_{a b c} \hat{\sigma}^{(c)}$. To interchange $x \rightarrow y$ one could do $\hat{\sigma}^{(x)} \rightarrow \hat{\sigma}^{(x)}$ and $\hat{\sigma}^{(y)} \rightarrow-\hat{\sigma}^{(y)}$. This would, however, result in $\hat{\sigma}^{(z)} \rightarrow-\hat{\sigma}^{(z)}$ in order to satisfy the Pauli commutation relations. So $x$ and $y$ cannot be interchanged without changing the sign of $z$. This also results in the same coordinate system and thus no interchange was made in the end. As such there is no symmetry to transform $x$ into $y$, explaining why a sign difference can be present.

\section{SUSCEPTIBILITY}

A divergence in the susceptibility indicates the existence of a phase transition. To calculate the susceptibility we use the scheme presented in [20] and apply a field in the $x y$ plane, which corresponds to adding a term, (11), to the system Hamiltonian. The resulting magnetization is given by

$$
\vec{M}(h, \theta)=\left(\begin{array}{ll}
\chi_{x x} & \chi_{x y} \\
\chi_{y x} & \chi_{y y}
\end{array}\right)\left(\begin{array}{l}
h \cos (\theta) \\
h \sin (\theta)
\end{array}\right)
$$

The susceptibility tensor can be extracted through

$$
\chi_{\alpha \beta}=\left.\frac{\partial M_{\alpha}}{\partial h_{\beta}}\right|_{h=0},
$$

with $M_{\alpha}$ the magnetization in the $x$ or $y$ direction. To obtain a scalar value the average angular susceptibility can be
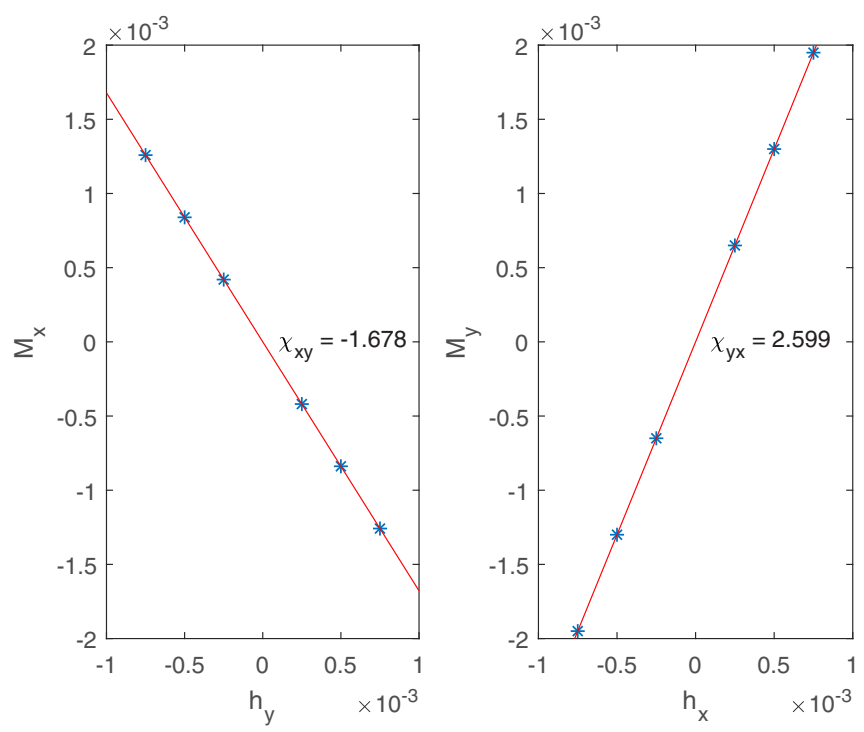

FIG. 6. Exact solution of the magnetization (blue asterisks) of system $M_{x}$ in the $x$ direction (left) and $M_{y}$ in the $y$ direction (right) under the influence of a small magnetic field in the $y$ and $x$ directions, respectively, for a $3 \times 3$ lattice at coupling strength $J_{y}=1.25 \gamma$. This result is obtained through solving the master equation. The magnetization as a function of the applied field is given by a linear function (red line); this means that $\chi_{\alpha \beta}$ is given by the slope of this linear function because of (18). As is clear in both panels, $\chi_{x y}$ and $\chi_{y x}$ have different signs, and furthermore, we find that $\left|\chi_{x y}\right| \neq\left|\chi_{y x}\right|$.

calculated as follows:

$$
\chi_{\mathrm{av}}=\left.\frac{1}{2 \pi} \int_{0}^{2 \pi} d \theta \frac{\partial|\vec{M}(h, \theta)|}{\partial h}\right|_{h=0} .
$$

For a more elaborate discussion we refer to [20].

When we calculate the susceptibility tensor for different cluster and lattice sizes we find that $\chi_{x y} \neq \chi_{y x}$. This is strikingly different from the case of closed systems, where the susceptibility is found from the free energy $F, \chi_{x y}=\frac{\partial^{2} F}{\partial h_{x} \partial h_{y}}=$ $\frac{\partial^{2} F}{\partial h_{y} \partial h_{x}}=\chi_{y x}$. We find this result even in the mean-field approximation. If we take the parameter values $J_{x}=J_{y}=J_{z}$ we find that $\chi_{x y}=-\chi_{y x}$ from (16). Numerical results show that for general coupling parameters the magnitudes differ and in general we find $\left|\chi_{x y}\right| \neq\left|\chi_{y x}\right|$ and reciprocity is broken. This is shown in Fig. 6 by solving the master equation with an applied field for a $3 \times 3$ lattice at coupling strength $J_{y}=0.25 \gamma$. In closed quantum systems the existence of the free energy and the ability to interchange the partial derivatives impose a symmetry on the system that need not be explicitly present in the system (e.g., a system where $J_{x} \neq J_{y} \neq J_{z}$ ). This result shows that the lack of a free energy in open quantum systems means no such symmetry is imposed.

For the 2D lattices we have two regions of interest: the earlier mentioned right-hand region, studied in [22], [23], and [43]; and the left-hand region, discussed in Sec. IV. In Fig. 7 the exact solution of the susceptibility for a $2 \times 2$ and a $3 \times 3$ lattice is shown. They were found by solving the master equation. Larger lattices are computationally not feasible and a more sophisticated method would have to be used, such as 


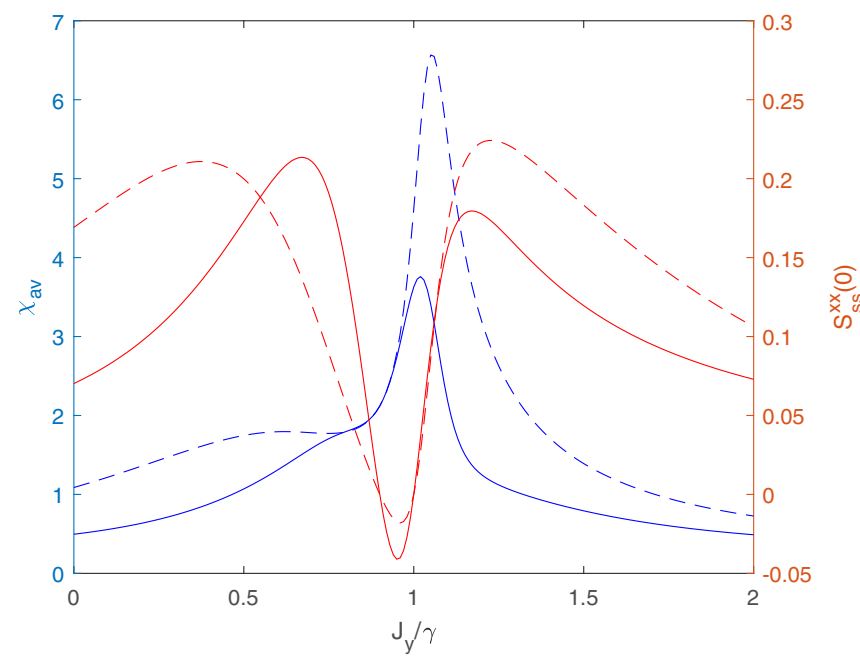

FIG. 7. Exact solution of the average angular susceptibility (bottom two, blue lines) and steady-state spin structure factor (top two, red lines) of a $2 \times 2$ (solid line) and a $3 \times 3$ (dashed line) lattice as a function of $J_{y}$, obtained through solving the master equation. Both solutions show a 'shoulder' for $J_{y}<0.9 \gamma$. This shoulder could be a second peak in the susceptibility, masked by the higher peak on the right, suggesting a phase transition. The position of this peak corresponds to the nonzero region in the steady-state spin structure factor.

the corner space method [20]. The right-hand region of the susceptibility has been studied in [20]. The presence of a peak in the susceptibility could indicate a phase transition. Note the 'shoulder' which is present on the left side. This could indicate the presence of a second peak, partially masked by the higher peak on the right-hand side. These two peaks move away from each other when the lattice size is increased. The peak on the left, however, is not sharp and it remains to be seen whether it diverges for larger lattice sizes and whether a true phase transition is present. It should be noted that the positions of the shoulder do coincide with a sharp decrease in the steady-state spin structure factor. These findings show the possible presence of a phase transition which is completely missed when the quantum correlations are neglected.

\section{CONCLUSIONS}

We study the dissipative $X Y Z$ Heisenberg model with the cluster Gutzwiller Monte Carlo. This method allows for the inclusion of short-range quantum correlations as well as classical spatial correlations. Calculation of the steady-state spin structure factor shows the appearance of a ferromagnetic region and two paramagnetic regions also found in Ref. [25] and Ref. [43]. We show the possible existence of another ferromagnetic phase which is completely missed when quantum correlations are neglected. A calculation of the susceptibility tensor shows how reciprocity is broken, a feature not observed in closed quantum systems. Moreover, increasing the magnetic field suppresses the magnetization; this is also in contrast with closed quantum systems. We show that the inclusion of short-range quantum correlations causes the sharpening of the crossover between the ferromagnetic and the paramagnetic region to occur for smaller lattice sizes. Even though short-range quantum correlations have a big impact on the phase diagram of the system, it must be noted that they only qualitatively match the exact results and long-range quantum correlations play an important role in the dynamics of the system.

\section{ACKNOWLEDGMENTS}

Discussions with C. Ciuti, F. Storme, W. Verstraelen, and M. Van Regemortel are gratefully acknowledged. This work was supported by BOF DOCPRO Universiteit Antwerpen. Some of the computational resources and services used in this work were provided by the VSC (Flemish Supercomputer Center), funded by the Research Foundation-Flanders (FWO) and the Flemish Government department EWI.
[1] I. Bloch, J. Dalibard, and W. Zwerger, Rev. Mod. Phys. 80, 885 (2008).

[2] I. Carusotto and C. Ciuti, Rev. Mod. Phys. 85, 299 (2013).

[3] M. J. Hartmann, J. Opt. 18, 104005 (2016).

[4] C. Noh and D. G. Angelakis, Rep. Prog. Phys. 80, 016401 (2017).

[5] S. R. K. Rodriguez, W. Casteels, F. Storme, N. Carlon Zambon, I. Sagnes, L. Le Gratiet, E. Galopin, A. Lemaitre, A. Amo, C. Ciuti, and J. Bloch, Phys. Rev. Lett. 118, 247402 (2017).

[6] M. Fitzpatrick, N. M. Sundaresan, A. C. Y. Li, J. Koch, and A. A. Houck, Phys. Rev. X 7, 011016 (2017).

[7] K. Baumann, C. Guerlin, F. Brennecke, and T. Esslinger, Nature (London) 464, 1301 (2010).

[8] K. Baumann, R. Mottl, F. Brennecke, and T. Esslinger, Phys. Rev. Lett. 107, 140402 (2011).

[9] F. Brennecke, R. Mottl, K. Baumann, R. Landig, T. Donner, and T. Esslinger, Proc. Natl. Acad. Sci. U.S.A. 110, 11763 (2013).

[10] J. M. Fink, A. Dombi, A. Vukics, A. Wallraff, and P. Domokos, Phys. Rev. X 7, 011012 (2017).
[11] T. Fink, A. Schade, S. Höfling, C. Schneider, and A. Imamoglu, Nat. Phys. 14, 365 (2018).

[12] W. Casteels, R. Fazio, and C. Ciuti, Phys. Rev. A 95, 012128 (2017).

[13] M.-J. Hwang, P. Rabl, and M. B. Plenio, Phys. Rev. A 97, 013825 (2018).

[14] U. Marzolino and T. Prosen, Phys. Rev. B 96, 104402 (2017).

[15] A. Tomadin, V. Giovannetti, R. Fazio, D. Gerace, I. Carusotto, H. E. Türeci, and A. Imamoglu, Phys. Rev. A 81, 061801(R) (2010).

[16] A. Le Boité, G. Orso, and C. Ciuti, Phys. Rev. Lett. 110, 233601 (2013)

[17] S. Diehl, A. Tomadin, A. Micheli, R. Fazio, and P. Zoller, Phys. Rev. Lett. 105, 015702 (2010).

[18] A. Tomadin, S. Diehl, and P. Zoller, Phys. Rev. A 83, 013611 (2011).

[19] A. Biella, F. Storme, J. Lebreuilly, D. Rossini, R. Fazio, I. Carusotto, and C. Ciuti, Phys. Rev. A 96, 023839 (2017). 
[20] R. Rota, F. Storme, N. Bartolo, R. Fazio, and C. Ciuti, Phys. Rev. B 95, 134431 (2017).

[21] V. R. Overbeck, M. F. Maghrebi, A. V. Gorshkov, and H. Weimer, Phys. Rev. A 95, 042133 (2017).

[22] T. E. Lee, S. Gopalakrishnan, and M. D. Lukin, Phys. Rev. Lett. 110, 257204 (2013).

[23] J. Jin, A. Biella, O. Viyuela, L. Mazza, J. Keeling, R. Fazio, and D. Rossini, Phys. Rev. X 6, 031011 (2016).

[24] F. Nissen, S. Schmidt, M. Biondi, G. Blatter, H. E. Türeci, and J. Keeling, Phys. Rev. Lett. 108, 233603 (2012).

[25] J. Jin, D. Rossini, R. Fazio, M. Leib, and M. J. Hartmann, Phys. Rev. Lett. 110, 163605 (2013).

[26] E. T. Owen, J. Jin, D. Rossini, R. Fazio, and M. J. Hartmann, New J. Phys. 20, 045004 (2018).

[27] F. A. Y. N. Schröder and A. W. Chin, Phys. Rev. B 93, 075105 (2016).

[28] M. T. Manzoni, D. E. Chang, and J. S. Douglas, Nat. Commun. 8, 1743 (2017).

[29] R. Orús and G. Vidal, Phys. Rev. B 78, 155117 (2008).

[30] F. Verstraete, J. I. Cirac, and V. Murg, Adv. Phys. 57, 143 (2008).

[31] U. Schollwoeck, Ann. Phys. 326, 96 (2011).

[32] E. Mascarenhas, H. Flayac, and V. Savona, Phys. Rev. A 92, 022116 (2015).

[33] J. Cui, J. I. Cirac, and M. C. Bañuls, Phys. Rev. Lett. 114, 220601 (2015).

[34] F. Verstraete, J. J. García-Ripoll, and J. I. Cirac, Phys. Rev. Lett. 93, 207204 (2004).

[35] M. Zwolak and G. Vidal, Phys. Rev. Lett. 93, 207205 (2004).

[36] S. Finazzi, A. Le Boité, F. Storme, A. Baksic, and C. Ciuti, Phys. Rev. Lett. 115, 080604 (2015).

[37] H. Weimer, Phys. Rev. Lett. 114, 040402 (2015).
[38] V. R. Overbeck and H. Weimer, Phys. Rev. A 93, 012106 (2016).

[39] D. P. S. McCutcheon, N. S. Dattani, E. M. Gauger, B. W. Lovett, and A. Nazir, Phys. Rev. B 84, 081305(R) (2011); 84, 119903(E) (2011).

[40] F. A. Pollock, D. P. S. McCutcheon, B. W. Lovett, E. M. Gauger, and A. Nazir, New J. Phys. 15, 075018 (2013).

[41] N. Suri, F. C. Binder, B. Muralidharan, and S. Vinjanampathy, Eur. Phys. J. Special Topics 227, 203 (2018).

[42] W. Casteels and M. Wouters, Phys. Rev. A 95, 043833 (2017).

[43] W. Casteels, R. M. Wilson, and M. Wouters, Phys. Rev. A 97, 062107 (2018).

[44] H. Pichler, J. Schachenmayer, A. J. Daley, and P. Zoller, Phys. Rev. A 87, 033606 (2013).

[45] W. Verstraelen and M. Wouters, Appl. Sci. 8, 1427 (2018).

[46] E. Mascarenhas, arXiv:1712.00987 [quant-ph].

[47] A. Nagy and V. Savona, Phys. Rev. A 97, 052129 (2018).

[48] A. Kshetrimayum, H. Weimer, and R. Orús, Nat. Commun. 8, 1291 (2017).

[49] E. B. Davies, Commun. Math. Phys. 15, 277 (1969).

[50] A. Barchielli and V. P. Belavkin, J. Phys. A 24, 1495 (1991).

[51] J. Dalibard, Y. Castin, and K. Mølmer, Phys. Rev. Lett. 68, 580 (1992).

[52] R. Dum, P. Zoller, and H. Ritsch, Phys. Rev. A 45, 4879 (1992).

[53] K. Mølmer, Y. Castin, and J. Dalibard, J. Opt. Soc. Am. B 10, 524 (1993).

[54] H. Carmichael, An Open Systems Approach to Quantum Optics, Vol. $m 18$, Lecture Notes in Physics (Springer-Verlag, Berlin, 1993).

[55] H. J. Carmichael, Phys. Rev. Lett. 70, 2273 (1993).

[56] H.-P. Breuer and F. Petruccioni, The Theory of Open Quantum Systems (Oxford University Press, New York, 2002).

[57] M. C. Gutzwiller, Phys. Rev. Lett. 10, 159 (1963). 